\title{
STRUCTURE OF LANDMARK LOOP \\ RIBONUCLEOPROTEIN MATRICES IN PLEURODELES WALTLII LAMPBRUSH CHROMOSOMES VISUALIZED BY SCANNING ELECTRON MICROSCOPY
}

\author{
M. L. BONNANFANT-JAÏS, E. N'DA, M. PENRAD-MOBAYED \\ AND N. ANGELIER \\ Centre de Biologie Cellulaire, CNRS, 67, rue Maurice Günsbourg, 94200 Ivry-sur-Seine, \\ France
}

\section{SUMMARY}

Landmark loop ribonucleoprotein (RNP) matrices of Pleurodeles waltii lampbrush chromosomes were systematically examined by scanning electron microscopy (SEM). The results, which corroborated similar studies by electron microscopy (EM), showed that RNP transcripts in normal loops, and RNP matrices in granular, globular and dense loops, are composed of one basic structure: an RNP particle with a diameter of $30 \mathrm{~nm}$.

SEM observations also clarified the spatial arrangement of this particle in the RNP matrices of all the loop types examined.

The specific morphology of normal, granular, globular and dense loop RNP matrices depended on the degree of compaction of the transcription products; this compaction resulted both from the packaging of RNP transcripts and the progressive coiling of the loop axis.

\section{INTRODUCTION}

A recent electron microscopic study of the different kinds of loops of Pleurodeles waltii lampbrush chromosomes provided new information about the structure of the loop RNP matrix (N'Da et al. 1986). This study clearly revealed that the fundamental structure of both normal and landmark loop ribonucleoprotein (RNP) matrices consists of a basic RNP particle some $30 \mathrm{~nm}$ in diameter.

As already described in lampbrush chromosomes of Pleurodeles and other species, each transcript along the DNA axis of the normal loops consists of such particles, which are interconnected (Miller, 1966; Malcolm \& Sommerville, 1977; Mott \& Callan, 1975; Spring \& Franke, 1981; N'Da et al. 1986).

In Pleurodeles lampbrush chromosomes, dense aggregates whose size increases along the loop axis, form the matrices of granular, globular and dense loops. These matrices are composed of $30 \mathrm{~nm}$ RNP particles that are aggregated tightly, making the matrices increasingly compact (N'Da et al. 1986).

The scanning electron microscopic (SEM) technique allowed us to envisage systematic study of all types of loop RNP matrices (Angelier, Paintrand, Lavaud \& Lechaire, 1984). Furthermore, identification of the bivalents is easy in SEM at low

Key words: amphibian, lampbrush chromosomes, landmarks loops, scanning electron microscopy, RNP matrix. 

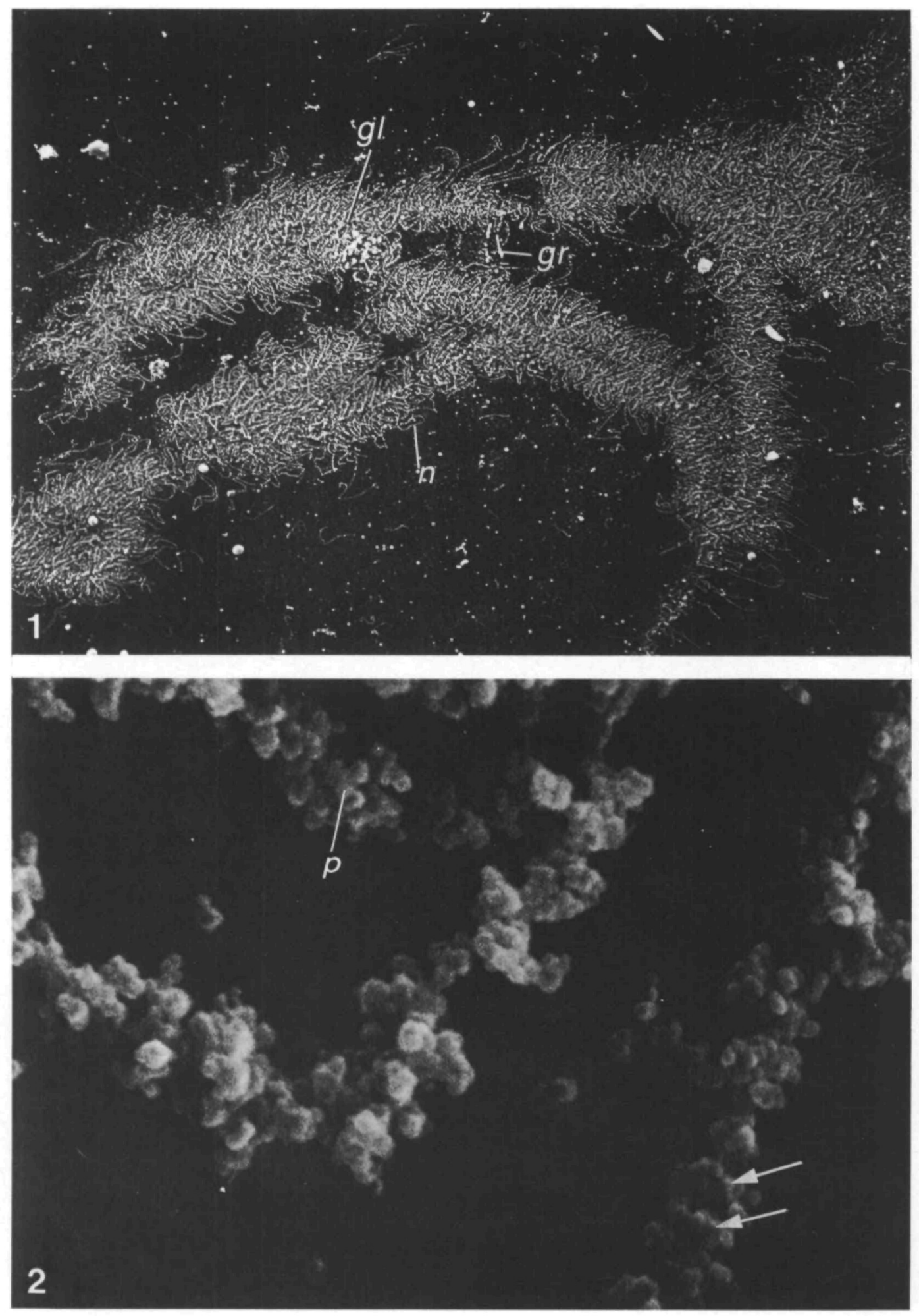
magnification but these chromosomes can also be observed at high magnification, equivalent to that used in transmission electron microscopy (TEM); this permits the correlation of these two aspects of the fine organization of lampbrush chromosomes.

In this respect the present SEM study provides new precise information concerning the spatial arrangement of the RNP matrices of the different kinds of loops.

\section{MATERIALS AND METHODS}

\section{Chromosome preparations}

In this study we used female newts of the Pleurodeles waltlii species (Amphibia and Urodela). Ovaries were removed from newts that had been anaesthetized in $0.1 \%$ MS 222 (Sandoz). Germinal vesicles of large oocytes were isolated by hand in a physiological medium containing $75 \mathrm{~mm} \cdot \mathrm{KCl}, 25 \mathrm{~mm}-\mathrm{NaCl}$ buffered to $\mathrm{pH} 7 \cdot 2$ with $10 \mathrm{~mm}$ - Tris $\cdot \mathrm{HCl}, 0.01 \mathrm{~mm}-\mathrm{MgCl}_{2}$ and $0.01 \mathrm{~mm}$ $\mathrm{CaCl}_{2}$ (Gall, 1954). Single clean nuclei were transferred to individual centrifugation chambers containing medium and consisting of a circular glass slide with a central hole sealed by a round coverslip (diameter, $12 \mathrm{~mm}$ ) glued to the underside of the slide. In the chamber, the nuclear envelope was removed with needles and forceps, and the nuclear content was centrifuged at $1500 \mathrm{~g}$ for $30 \mathrm{~min}$ onto the coverslip sealing the chamber. After centrifugation, the chromosomes were observed in phase contrast with a Carl Zeiss inverted microscope. Bivalents were identified, photomicrographed, and processed for SEM.

\section{Scanning electron microscopy}

During fixation and postfixation, the chromosomes were kept in the centrifugation chamber. The lampbrush chromosomes were fixed for $20 \mathrm{~min}$ with $1 \%$ glutaraldehyde buffered to $\mathrm{pH} 7 \cdot 2$ in $0 \cdot 1 \mathrm{M}$-phosphate buffer. After postfixation with $1 \%$ osmium tetroxide, the coverslip was removed from the slide and chromosome preparations were dehydrated in a graded series of ethanol, then in a graded series of acetones containing absolute 'ethanol. The samples were dried at the critical point in a Balzers CPD 010 apparatus using liquid $\mathrm{CO}_{2}$ as the intermediate fluid. The specimens were immediately transferred to a vacuum evaporator (Edwards S15 PA), sputter coated and maintained at a pressure of $10^{-1}$ Torr for several minutes. After evacuation, purified argon was introduced into the bell jar to a pressure of $7 \times 10^{-2}$ Torr. Samples were shadowed with gold for $45 \mathrm{~s}$ at $20 \mathrm{~mA}$ and examined with a Philips 505 scanning electron microscope.

\section{RESULTS}

\section{The normal loop RNP matrix}

At high magnification, the structure of the normal loop RNP matrix was immediately apparent; each transcript consisted of a string of RNP particles some $50 \mathrm{~nm}$ in diameter. These particles were remarkably uniform in size throughout the RNP matrix of all normal type loops. These SEM observations confirmed the linear arrangement of RNP matrix particles along the transcripts observed in EM. The difference in the size of the RNP particles seen by EM on thin sections and those

Fig. 1. Two of twelve bivalents of the $P$. waltii karyotype. Bivalents were easy to identify at low magnification. $g l$, globular loop; $g r$, granular loop; $n$, normal loop. $\times 300$.

Fig. 2. RNP matrix organization of a normal loop: each RNP transcript is composed of RNP particles of the same size $(50 \mathrm{~nm}$, including the $20 \mathrm{~nm}$ gold coat) and displays a helical arrangement around the loop axis (arrows); $p$, RNP particle. $\times 46200$. 
visualized by SEM (30 versus $50 \mathrm{~nm}$ ) corresponded to the gold coat with which structures were covered during sample shadowing for SEM.

SEM observations also supplied information about the spatial arrangement of the RNP matrix. RNP transcripts formed a thin helical sheath around the loop axis. The same spatial arrangement of RNP transcripts was observed all along the normal loops (Figs 1, 2).

\section{The granular loop RNP matrix}

Granular loops were characterized by an RNP matrix composed of granules of various sizes. Each loop displayed obvious polarity, since the size of the granules along the axis of the loop increased between its thin and thick insertions along the chromosomal axis. Granule size varied from one loop to another (Fig. 3A).

Examination of the different aspects of the granular loops showed that their RNP matrix organization gradually evolved from a normal to a globular type of matrix. In some granular loops, the thin insertion exhibited an RNP matrix similar to that of a normal loop and then progressively aggregated into small granules along the loop axis (Fig. 3A). Nearly all the granular loops were characterized by an RNP matrix composed either of small granules whose size increased along the loop axis from $500 \mathrm{~nm}$ to about $1 \mu \mathrm{m}$, or of larger granules $1-3 \mu \mathrm{m}$ in diameter (Fig. $3 \mathrm{~A}, \mathrm{~B}$ ). In the last case, the loop matrix exhibited a globular appearance at its thick insertion on the chromosomal axis (Fig. 3B).

At high magnification, each granule was seen to consist of RNP particles of uniform size, similar to those observed along the RNP transcripts of normal loops (Figs 4, 5). Around the loop axis, the granules retained the initial helical arrangement of the normal loop transcripts (Fig. 4B). The granules composing the loop RNP matrix obviously resulted from the aggregation of adjacent RNP transcripts.

As granule size increased along the loop, the loop axis gradually coiled, which explains the zig-zag arrangement of the granules observed on thin EM sections (N'Da et al. 1986). When granules were large, this coiling of the loop axis became very clear and granules were very close together (Fig. 5).

\section{The globular loop RNP matrix}

The RNP matrix of globular loops was much thicker than that of normal and granular loops, and was composed of globules $3-4 \mu \mathrm{m}$ in diameter (Fig. 6A).

Fig. 3. A,B. Different types of granular loops. A. Granule size varies along the granular loop axis, thus clearly showing the polarity of the RNP matrix. Granule size also varies from one loop to another. In this view, matrices of the different types of granular loops exhibit a morphological evolution starting from the organization of a normal type (thin arrow) to that of a globular type (thick arrow). $g r$, granular loop; $g l$, globular loop; $n$, normal loop; $\times 1750$. B. Two types of granular loops: the upper one is composed of small granules of about $500 \mathrm{~nm}$ in diameter, and the lower loop of larger granules $1-3 \mu \mathrm{m}$ in diameter. $\times 5750$. 

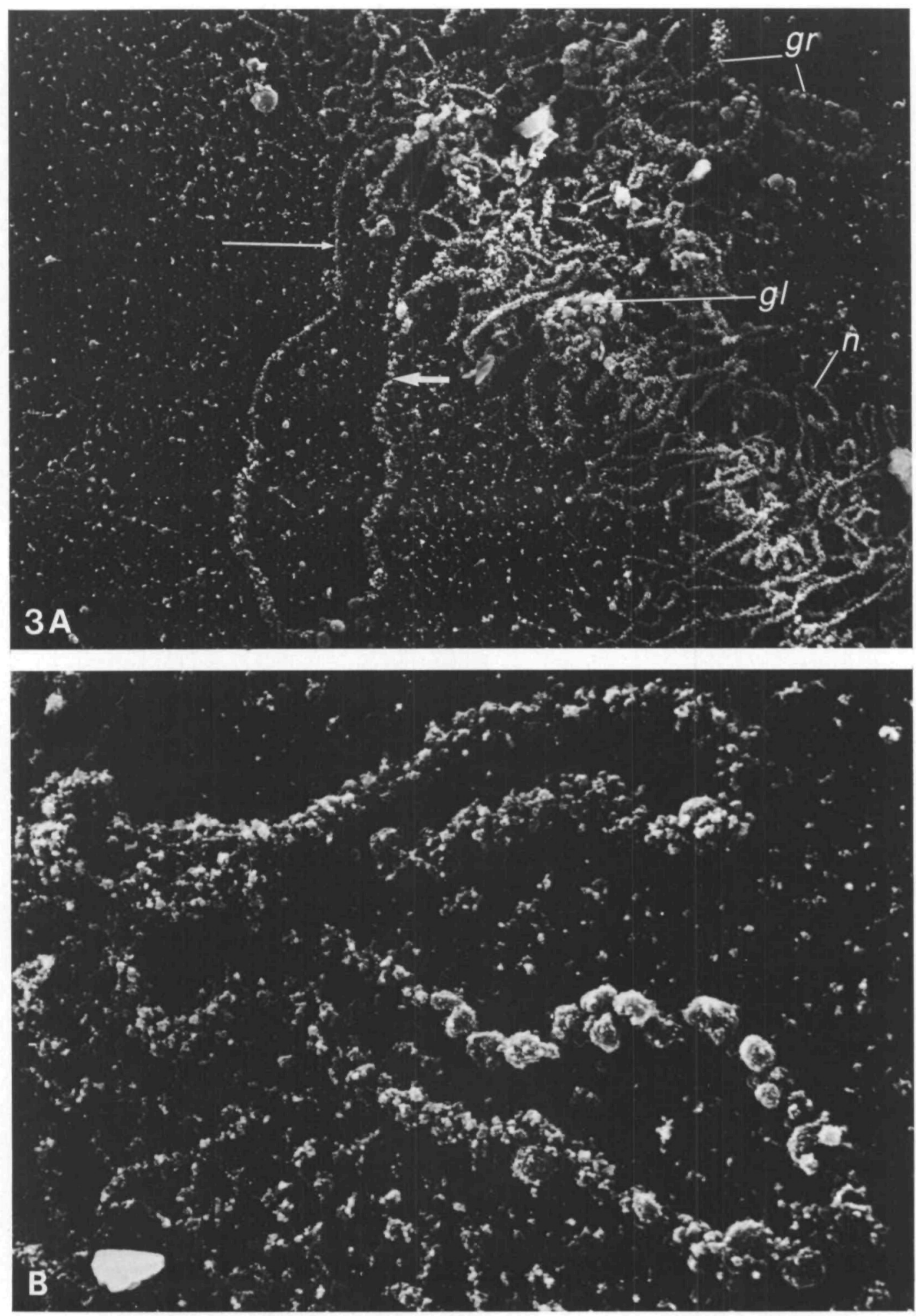
Each globule (i.e. matrix) comprised RNP particles with a diameter of $50 \mathrm{~nm}$ including the $20 \mathrm{~nm}$ gold coat (Fig. 6B); these particles resembled those already observed in the normal and granular types of RNP matrix. The presence of transcript interactions connecting the globules (Fig. 6B), suggested that these globules were formed by the tight packaging of adjacent transcripts.

Compared to the normal and granular loops, most of the globular loops did not unfold from the chromosomal axis. The coiling of the globular loop axis already observed for the granular loop was more pronounced, so that the globular loops formed a spiral (Fig. 7A). When the loop axis was tightly coiled, the globules tended to fuse and the RNP matrix formed a thick continuous sheath around the loop axis (Fig. 7B).

\section{The dense loop RNP matrix}

Dense matrix loops formed short compact lobes projecting from the chromosomal axis. The usual pattern of loop organization was not recognizable.

At high magnification, the RNP matrix of these dense loops consisted of RNP particles of exactly the same size as the particles of normal, granular and globular type matrices (Fig. 8).

Coiling of the axis was tightest in the dense loops, which exhibited either a twisted or a compact appearance; their RNP matrix formed a thick continuous sheath around the loop axis (Fig. 8).

\section{DISCUSSION}

\section{Basic structure of the RNP matrix}

All the types of loops in $P$. waltlii lampbrush chromosomes were systematically studied by SEM at the same high magnification as that used in EM. These SEM observations showed that all the RNP matrix particles in normal, granular, globular and dense loops were remarkably uniform in size. Whatever the morphology of the lateral loop matrix, its fundamental structure consisted of an RNP particle $50 \mathrm{~nm}$ in diameter, including the $20 \mathrm{~nm}$ gold coat. These observations corroborate those performed by EM on thin sections, indicating that in all types of loops the basic structure of the RNP matrix is a $30 \mathrm{~nm}$ RNP particle (N'Da et al. 1986).

Under our experimental conditions, the thickness of the gold coat applied to the samples was precisely evaluated at $20 \mathrm{~nm}$. Angelier et al. (1984) observed the same uniformity in the size of the RNP particles constituting the matrices of all the different kinds of loops. However, they evaluated the diameter of these particles at $100-200 \mathrm{~nm}$, compared to our value of $30 \mathrm{~nm}$. The difference in RNP particle size might be due to the erroneous evaluation of the gold coat by these authors, although theirs was thicker because their shadowing lasted longer.

Fig. 4. A,B. Granular loop. A. Each granule is composed of RNP particles with a diameter of $50 \mathrm{~nm}$ and is formed by the packaging of adjacent transcripts. $p$, RNP particle. $\times 42000$. B. Granules exhibit a helical arrangement around the loop axis. $\times 42000$. 

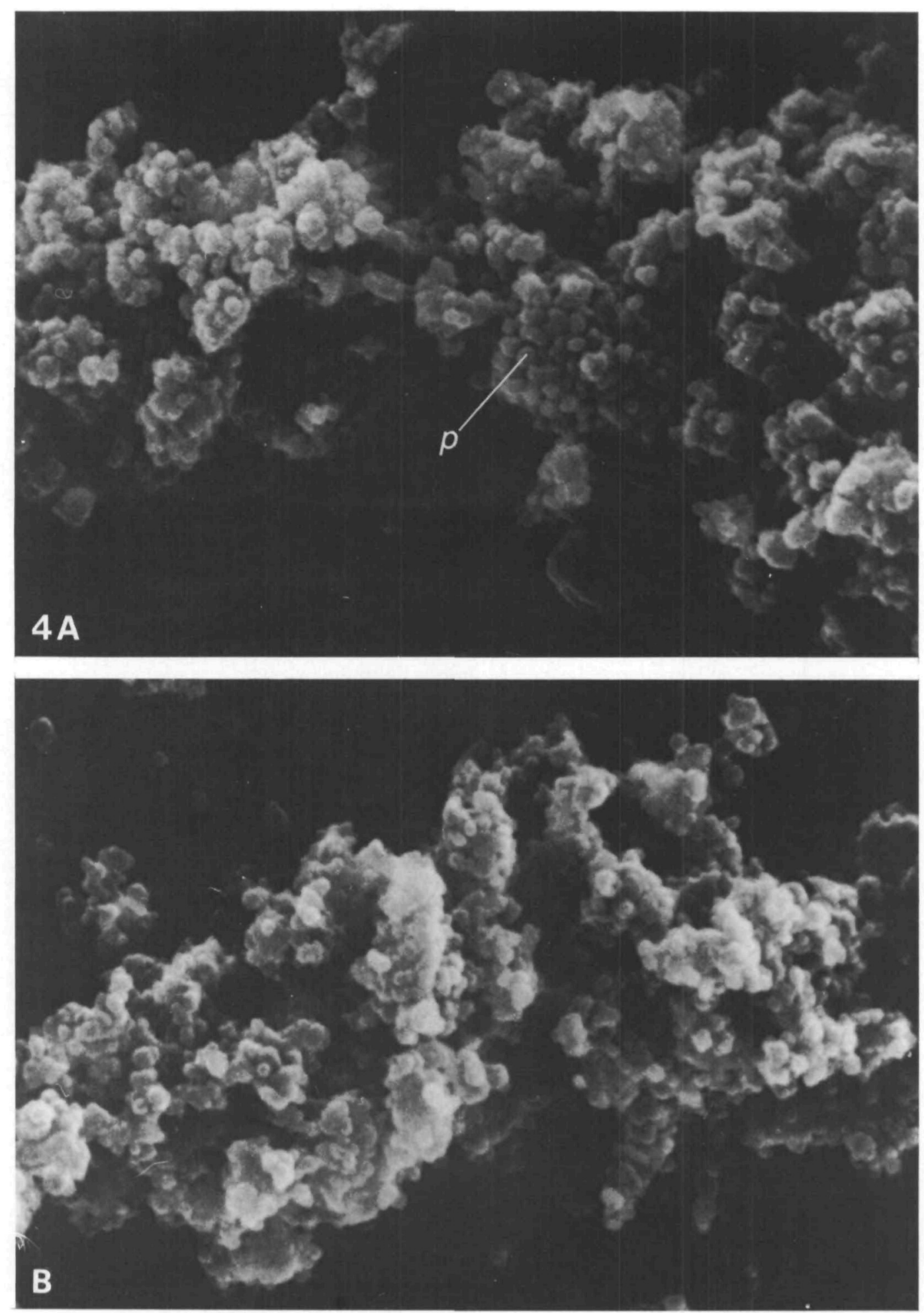

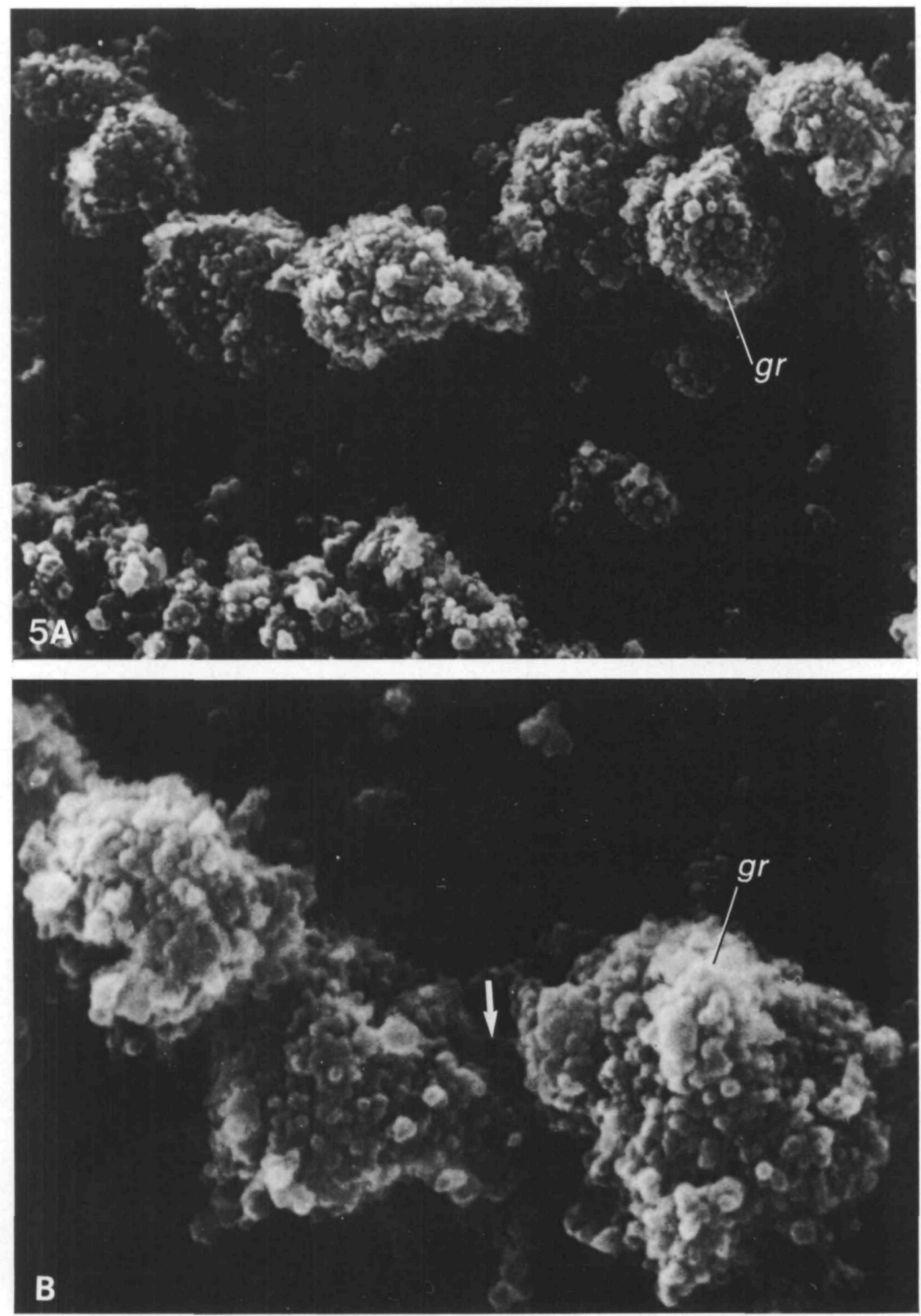

Fig. 5. For legend see p. 41 

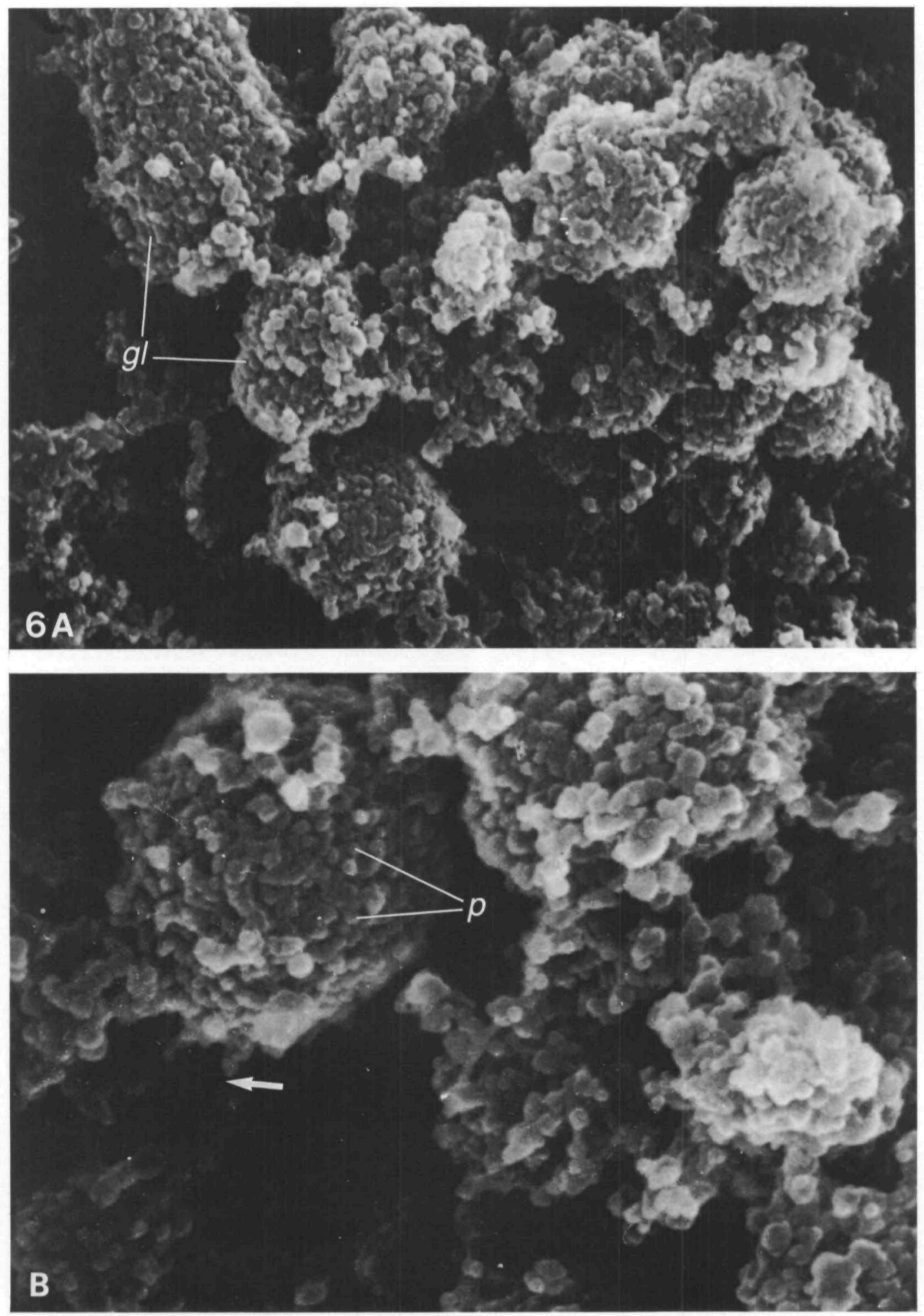

Fig. 6. For legend see p. 41 

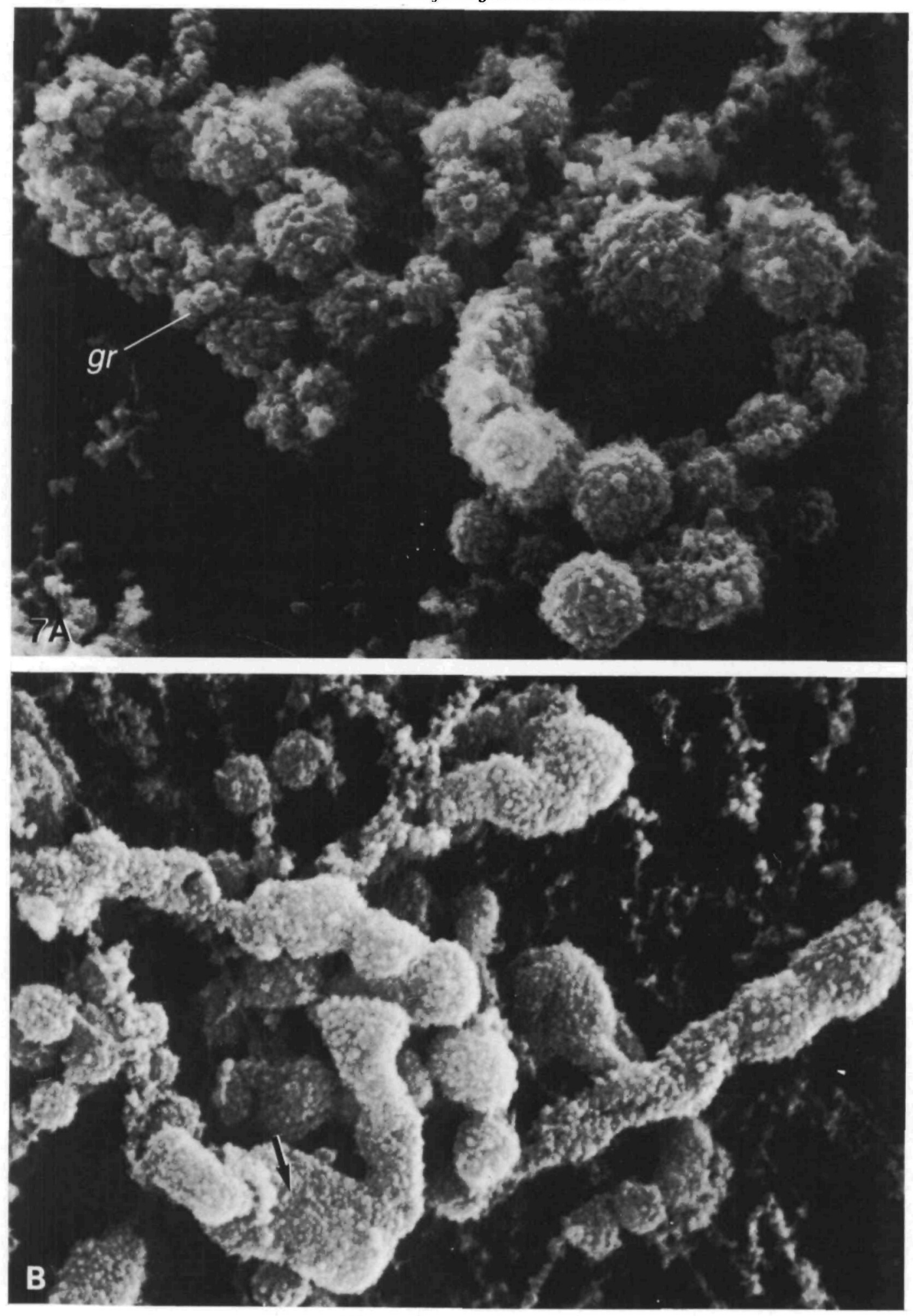

Fig. 7. For legend see p. 41 


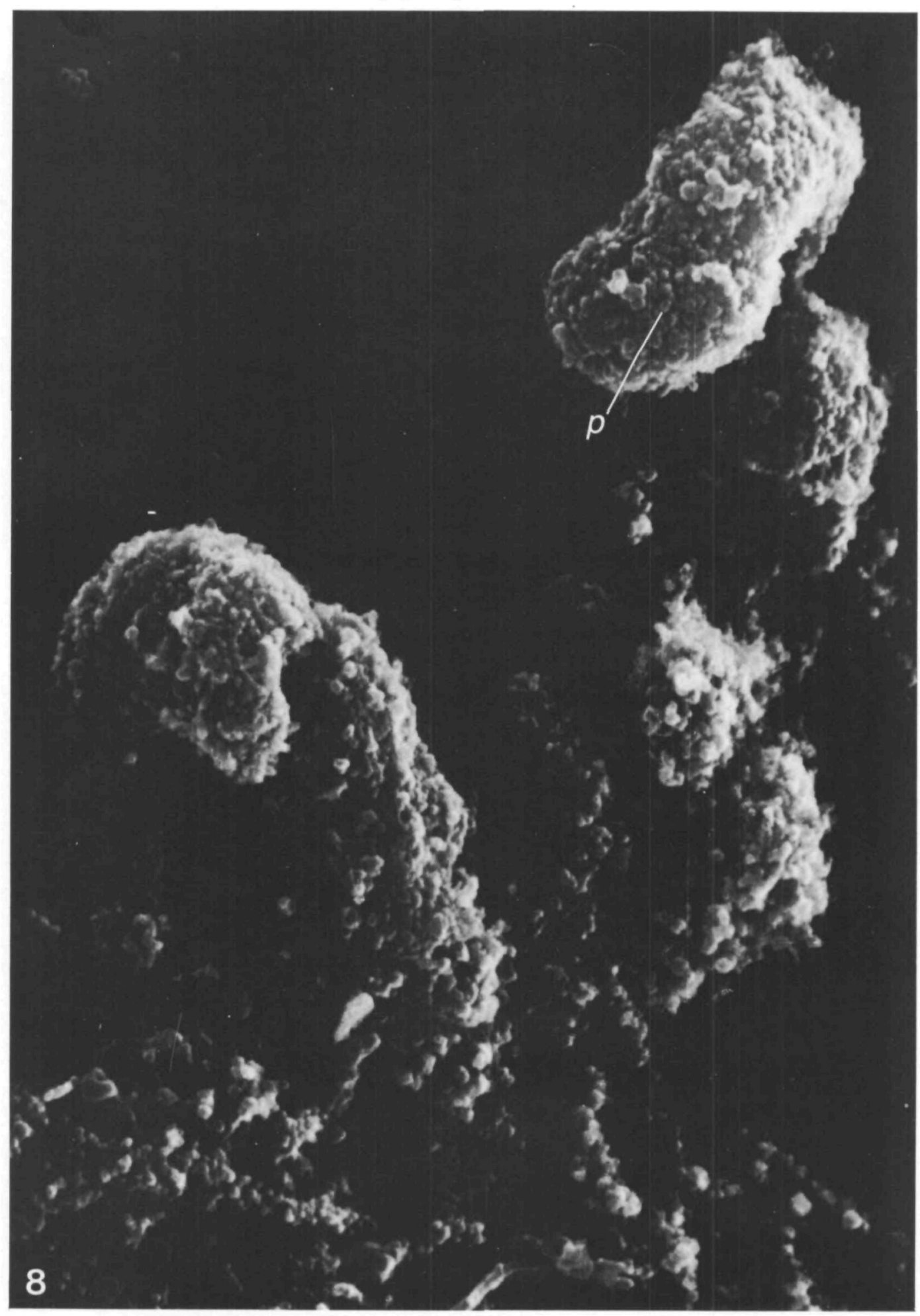

Fig. 8. For legend see p. 41 
Variable morphology of the loop matrix

In the different types of lateral loops, the thickness of the RNP matrix increased from normal to dense type loops and its organization grew denser as the transcripts aggregated to form granules, globules and finally a compact sheath.

SEM study of loop matrices allowed the spatial arrangement of transcription products along the loop axis to be defined, which is quite impossible in EM, even on thin serial sections.

9A

0

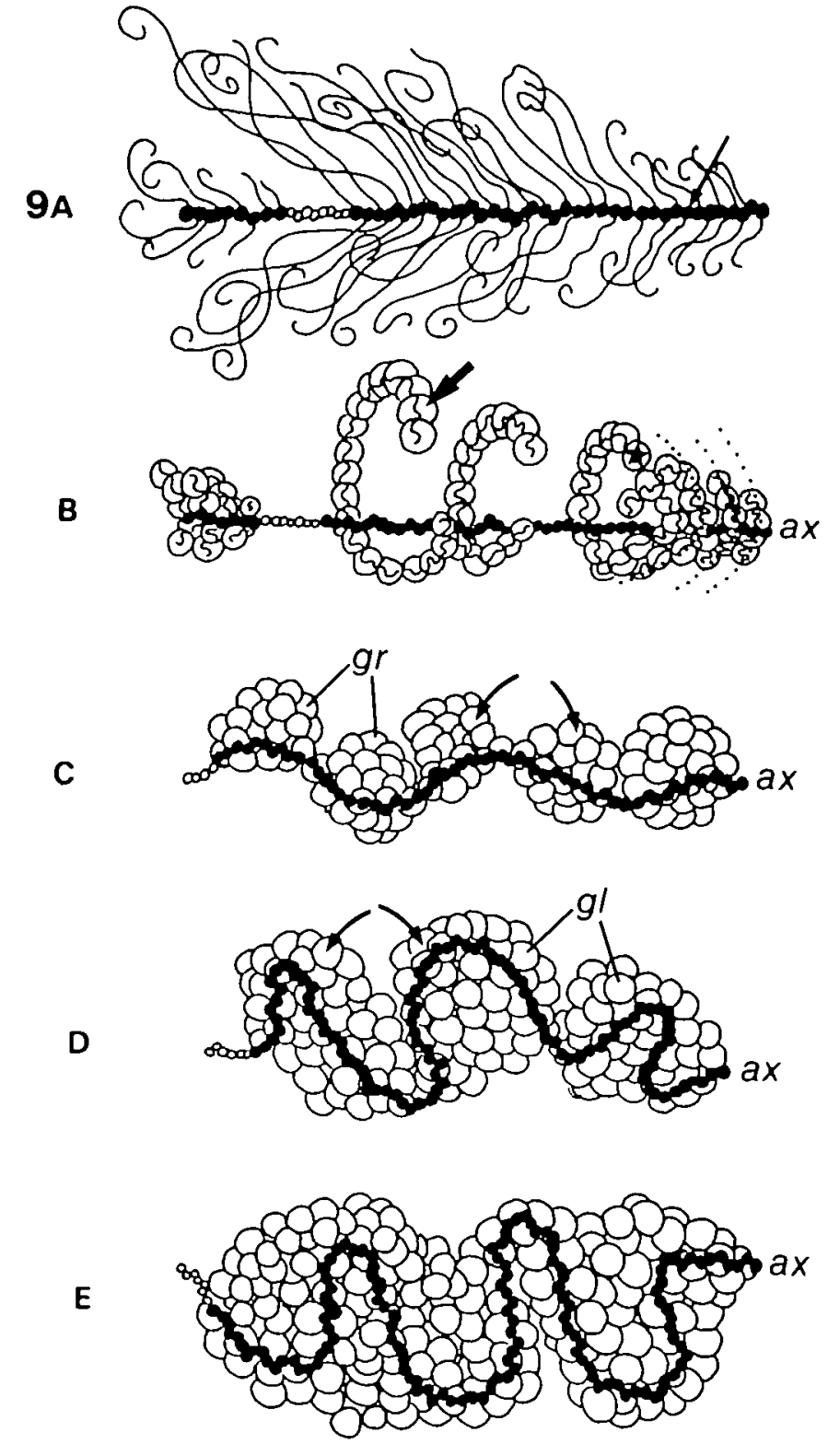


SEM observation of these loops provided good evidence that the morphological differences between them resulted from the various degrees of tightness in the packaging of the transcription products, as suggested by Angelier $e$ t al. (1984).

The aggregation of adjacent RNP transcripts might imply interactions between the constitutive proteins of the RNP units. This aggregation may cause coiling of the loop axis. Such coiling becomes more pronounced as the process of compaction involves RNP transcripts situated further and further apart. Examination of the different kinds of landmark loops shows that matrix components underwent gradual compaction. This implies both the progressive packaging of transcripts and concomitant gradual coiling of the loop axis; the fact that the aggregates grew progressively larger and that the dense loops exhibited intense compaction of their RNP matrix components corresponds to the observation of extremely tight coiling of their axis, schematically represented in Fig. 9.

Nevertheless, we cannot be sure that this morphological transformation observed on the landmark loops as a whole, corresponds to the transformation of a particular loop from a normal to a granular loop and then from a globular to a dense loop.

There are several arguments in favour of such a transformation. First, as we described, the granular type loop exhibited a morphological transformation from a normal type to a globular type loop. Second, we observed that in a few oocytes a certain type of landmark loop may be missing, at any stage of oogenesis; dense loops are the most striking example of such variability. However, because of the high

Fig. 5. A,B. Granular loop. A. As granules grow larger, the coiling of the loop axis becomes clearer. $g r$, granules. $\times 21000$. B. Transcript interactions between granules (arrow). $g r$, granule. $\times 42000$.

Fig. 6. A,B. Globular loop. A. The globular loop matrix is composed of globules about $3-4 \mu \mathrm{m}$ in diameter; polarity is no longer recognizable. $g l$, globule. $\times 17500$. B. Globules are composed of the same basic particle, with a diameter of $50 \mathrm{~nm}$. Several transcripts interactions (arrow) are clearly visible between the globules. $p$, RNP particle. $\times 46000$.

Fig. 7. A,B. Globular loops. A. The globular loop forms a spiral, characteristic of this type of lateral loop; $g r$, granular loop. $\times 16000$. B. In this view, globules have fused to form a continuous sheath around the loop axis (arrow). $\times 13000$.

Fig. 8. Dense matrix loop. Two aspects of dense loops: on the right a twisted matrix and on the left a thick compact matrix. The matrix of these dense loops is composed of particles $50 \mathrm{~nm}$ in diameter. $p$, RNP particle. $\times 21000$.

Fig. 9. A-E. Schematic representation of the progressive compaction of the RNP matrix and concomitant coiling of the loop axis. A. Transcription figure visualized on a lampbrush loop, spreads according to Miller's procedure. Thin arrow, RNA polymerase at the basis of each transcript. B. The normal loop RNP matrix. RNP transcripts (thick arrow) coil around the loop axis $(a x)$ in such a way that $30 \mathrm{~nm}$ RNP particles ( $\star$ ) of the transcripts show a helical arrangement (dotted lines). c. The granular loop RNP matrix. Interactions between RNP particles of adjacent transcripts induce their aggregation into granules $(g r)$. This transcript aggregation leads the loop axis $(a x)$ to curve in at the level of each granule. D. The globular loop RNP matrix. Aggregates of adjacent RNP transcripts grow progressively larger. The coiling of the loop axis $(a x)$ becomes more pronounced as the process of compaction involves RNP transcripts situated further and further apart. $g l$, globule. E. The dense loop RNP matrix. The compaction of the RNP transcripts is maximum. The matrix forms a continuous sheath around the loop axis (ax), which exhibits a concomitant extreme coiling. 
lateral-loop density, we were unable to establish clearly whether or not a normal loop develops instead of a landmark loop, at a precise site along the chromosomal axis. Third, globular loops were sometimes well developed but granular loops were missing, or the converse. This makes it all the more difficult to ascertain whether granular and globular loop formation constitute steps in the progressive compaction of the RNP matrix, since these processes always occur at virtually adjacent sites.

Further investigations are required to determine the individual transformation of such landmark loops. The variations in their morphology seen here might be connected with physiological variations in transcription.

\section{REFERENCES}

Angelier, N., Paintrand, M., Lavaud, A. \& Lechaire, J. P. (1984). Scanning electron microscopy of amphibian lampbrush chromosomes. Chromosoma 89, 243-253.

GaLL, J. G. (1954). Lampbrush chromosomes from oocyte nuclei of the newt. J. Morph. 94, 283-351.

Malcolm, D. B. \& Sommerville, J. (1977). The structure of nuclear ribonucleoprotein of amphibian oocytes. J. Cell Sci. 24, 143-165.

MilleR, O. L. (1966). Fine structure of lampbrush chromosomes. Nat. Cancer Inst. Monogr. 18, 79-99.

Motr, M. R. \& Callan, H. G. (1975). An electron microscope study of the lampbrush chromosomes of the newt Triturus cristatus. F. Cell Sci. 17, 241-261.

N'Da, E., Bonnanfant-Jaîs, M. L., Penrad-Mobayed, M. \& Angelier, N. (1986). Size uniformity of RNP matrix particles in loops of Pleurodeles waltlii lampbrush chromosomes visualized by electron microscopy. F. Cell Sci. 81, 17-27.

SPRING, H. \& FRANKE, W. W. (1981). Transcriptionally active chromatin in loops of lampbrush chromosomes at physiological salt concentrations as revealed by electron microscopy sections. Eur. F. Cell Biol. 24, 298-308.

(Received 23 fuly 1985 - Accepted 23 September 1985) 